OPEN ACCESS

Edited by:

Elisa Ferrando-May,

University of Konstanz, Germany

Reviewed by:

Philipp Kaldis,

Agency for Science, Technology and Research, Singapore

Bennett Van Houten,

University of Pittsburgh, USA

*Correspondence:

Michael M. Seidman

seidmanm@grc.nia.nih.gov

Specialty section This article was submitted to

Cancer Genetics,

a section of the journal

Frontiers in Genetics

Received: 18 February 2016 Accepted: 22 April 2016

Published: 09 May 2016

Citation:

Huang J, Gali H, Paramasivam M,

Muniandy P, Gichimu J, Bellani MA

and Seidman MM (2016) Single

Molecule Analysis of Laser Localized

Interstrand Crosslinks.

Front. Genet. 7:84

doi: 10.3389/fgene.2016.00084

\section{Single Molecule Analysis of Laser Localized Interstrand Crosslinks}

\author{
Jing Huang, Himabindu Gali, Manikandan Paramasivam, Parameswary Muniandy, \\ Julia Gichimu, Marina A. Bellani and Michael M. Seidman*
}

Laboratory of Molecular Gerontology, National Institute on Aging, National Institutes of Health, Baltimore, MD, USA

DNA interstrand crosslinks (ICLs) block unwinding of the double helix, and have always been regarded as major challenges to replication and transcription. Compounds that form these lesions are very toxic and are frequently used in cancer chemotherapy. We have developed two strategies, both based on immunofluorescence (IF), for studying cellular responses to ICLs. The basis of each is psoralen, a photoactive (by long wave ultraviolet light, UVA) DNA crosslinking agent, to which we have linked an antigen tag. In the one approach, we have taken advantage of DNA fiber and immunoquantum dot technologies for visualizing the encounter of replication forks with ICLs induced by exposure to UVA lamps. In the other, psoralen ICLs are introduced into nuclei in live cells in regions of interest defined by a UVA laser. The antigen tag can be displayed by conventional IF, as can the recruitment and accumulation of DNA damage response proteins to the laser localized ICLs. However, substantial difference between the technologies creates considerable uncertainty as to whether conclusions from one approach are applicable to those of the other. In this report, we have employed the fiber/quantum dot methodology to determine lesion density and spacing on individual DNA molecules carrying laser localized ICLs. We have performed the same measurements on DNA fibers with ICLs induced by exposure of psoralen to UVA lamps. Remarkably, we find little difference in the adduct distribution on fibers prepared from cells exposed to the different treatment protocols. Furthermore, there is considerable similarity in patterns of replication in the vicinity of the ICLS introduced by the two techniques.

Keywords: DNA damage, replication, interstrand crosslinks, single molecule, DNA fiber, laser

\section{INTRODUCTION}

After several decades of effort by many laboratories, we have a detailed understanding of the cellular biochemistry that removes single strand adducts and repairs single and double strand breaks (DSBs). Imaging strategies, particularly those based on immunofluorescence (IF) microscopy, have been particularly helpful in elucidating these pathways and the factors that drive them. Perhaps the most extensive application of IF has been in the study of DSB repair. Fortuitously, proteins that accumulate in the vicinity of breaks introduced by irradiation, replication fork breakage, or enzymatic cleavage, form foci readily visualized by IF. This feature has been widely exploited and has been instrumental in defining the collective of proteins known as the DNA damage response (DDR), the subject of an enormous, and expanding, literature (Ciccia and Elledge, 2010). The appearance of foci in response to DSBs provides an important experimental tool for studying the 
complexity of the DDR, the timing of accumulation of specific factors to foci, and the dependence of one factor on another for recruitment. Furthermore, although the breaks are not explicitly detected, the proteins of the DDR foci serve as surrogate markers of their presence (Rothkamm et al., 2015).

An additional, and very powerful, technology introduced by the Bonner lab, demonstrated that exposure to localized laser light could provoke the introduction of DSBs which activated the DDR (Rogakou et al., 1999). Analyses of the early marker of the DDR, the now famous phospho-histone, $\gamma-\mathrm{H} 2 \mathrm{AX}$, by IF, revealed a stripe of signal corresponding to the line of laser exposure across the nucleus. In effect, the experimentalist created the "focus" with the laser. This has proven to be an immensely popular approach to studying the protein dynamics induced by DSBs. $\gamma$-H2AX foci are commonly assumed to be indicators of breaks. However, these are not necessarily quantitative reporters of breaks, and it has been difficult to address fundamental questions regarding DSB density and distribution in the regions of interest (ROI).

The obvious power of these imaging strategies raises the possibility of their use in the study of lesions other than DSBs. However, other forms of DNA damage are not as cooperative. Ultraviolet light introduces covalent adducts that, although they attract repair components of the DDR, do not directly stimulate the formation of discrete foci (see below). Similarly, many other DNA adducts do not induce DDR foci except as they block DNA replication. In those situations the disrupted replication fork is the actual instigator of the DDR. In order to follow the cellular response to the lesion, the problem posed by the absence of foci can be overcome by taking advantage of techniques to create foci. These approaches introduce adducts, in a restricted nuclear region, at a density that is higher than in the surrounding area. The accumulation of responsive proteins to these "artificial" foci can then be monitored. This has been possible with UV photoproducts by masking cells with micropore filters which block the passage of short wave UV. Only light passing through an occasional vertical pore reaches the cell layer. This results in nuclei with patches of photoproducts that can be detected and displayed by IF with photoproduct specific antibodies. Protein recruitment to the localized photoproducts can be monitored as well (Volker et al., 2001). This approach is limited to studies of adducts whose formation is dependent on light that can be blocked by the filters, typically ultraviolet photoproducts.

Analogous to the work with DSBs, it is also possible to use lasers of appropriate wavelength to introduce, in defined ROI in nuclei, adducts that are generated by photochemistry. UV photoproducts can, of course, be formed directly (Dinant et al., 2007), while exposure of DNA to radicals produced by absorption of laser energy by endogenous photosensors can produce oxidative lesions and/or single strand breaks (Lan et al., 2004).

Another DNA modification that is of great current interest is the interstrand crosslink (ICL). Agents that form them are highly toxic and are frequently used in cancer chemotherapy. ICLs present a potent challenge to replication and transcription as they are absolute blocks to unwinding of DNA. Consistent with their linkage of two strands, ICLs are repaired in two cycles of repair. In the first the two strands are uncoupled from one another in a process termed "unhooking" (Dronkert and Kanaar, 2001). This can be done either by endonucleolytic incisions on one strand on either side of an ICL, or by introduction of a nick in one of the strands followed by exonucleolytic digestion past the ICL (Sengerova et al., 2012). In both scenarios the "other strand" retains the resultant single strand adduct, termed the "crosslink remnant." After gap filling, necessarily involving a lesion bypass step, the crosslink remnant is removed by conventional nucleotide excision repair. Although this general scheme has been appreciated for many years, it has become apparent that there are many different proteins involved, and multiple pathways, most incompletely understood.

The field of ICL repair has been greatly stimulated by the establishment of an in vitro system, based on Xenopus egg extracts, that supports replication, and repair of a plasmid carrying a site specific ICL (Raschle et al., 2008). The very elegant work from the Walter lab has captured the imagination of the field and framed much of the current discussion. However, while a very productive experimental approach, analyses of ICL repair with plasmid based strategies are much influenced by the particulars of the assays. Furthermore, the events modeled by the plasmid systems represent a subset of those at the genomic level. Constructs that measure repair by relief of transcription suppression, of necessity have strong promoters and thus report features of transcription coupled repair of the ICLs (Hlavin et al., 2010). Plasmids that are templates for replication display events related to repair of ICLs in the context of replication fork encounters (Shen et al., 2009; Knipscheer et al., 2012). In order to parse the complexity of ICL repair in the mammalian genome other approaches are required.

We have developed two strategies, based on fluorescence imaging, for addressing questions about the encounter of replication forks with, and the cellular response to, genomic ICLs. At the heart of our experiments is a crosslinking compound whose adducts can be detected immunologically. This enables display of ICLs, and events associated with them, by various imaging techniques. At the outset of our studies we considered the experimental utility of clinically important crosslinking agents. Although Mitomycin C and cis-platinum are well established in cancer chemotherapy, they form a minority of ICLs as a fraction of total adducts $(<10 \%)$, which are not easily detected (Muniandy et al., 2010).

By contrast, the photoactive psoralens can form a very high frequency of ICLs (Lai et al., 2008). The requirement for photoactivation by long wave ultraviolet light (UVA) allows ICL formation to be controlled as to time and location. Furthermore, although psoralens cannot be imaged directly, they can be linked to detection tags. These are moieties that can be imaged directly, such as fluorescent proteins (Chalfie et al., 1994). Another example would be the FLAG peptide which can be detected by antibodies (Knappik and Pluckthun, 1994), or biotin which is bound by streptavidin (Nerurkar et al., 1984). Appropriately conjugated tags, including biotin and fluorescent molecules, can be participants in coupling reactions such as those employed in click chemistry, which has been applied in many areas of biological research (Kolb et al., 2001). As described below, we synthesized a psoralen linked to digoxigenin, a plant sterol not 
found in mammalian cells, and widely used as an immunotag (Miller et al., 1993).

In one approach, we combined immuno-quantum dot detection of single molecules with DNA fiber technology (Schwab and Niedzwiedz, 2011). We were able to visualize replication tracts in the vicinity of individual psoralen ICLs generated by photoactivation with a UVA lamp (Huang et al., 2013; Rohleder et al., 2016). In the other development, we used a confocal microscope equipped with a UVA laser to introduce psoralen ICLs into ROI in nuclei of live cells. The localized ICLs induce the recruitment of repair and DDR proteins (see below).

The antigen tag on the psoralen allows display of psoralen ICLs, by IF, in a laser photoactivated ROI. Although very useful for monitoring the repair of psoralen ICLs it is not possible, given the limit of resolution of confocal microscopes, to assess the density of ICLs along a DNA molecule within a ROI. In contrast, the single molecule resolution of the fiber/quantum dot technology readily permits determination of ICL frequency and distribution on an individual DNA strand. Thus, an important feature of our DNA fiber experiments is unavailable in the studies with the laser localized ICLs. As lasers can provide photoactivating light at much higher intensity than UVA lamps, it is possible that there are much higher lesion densities in the one technique than the other. Consequently, it is not certain that results with one light source could be directly compared with those of the other, particularly as regards replication in the laser defined ROIs.

In the experiments reported here, we illustrate applications of laser localization and immuno-quantum dot imaging of ICLs. We have used the latter technology to answer fundamental questions about the frequency and distribution of the laser localized ICLs in the ROI. In addition, we directly address the issue of replication in the vicinity of laser induced ICLs.

\section{MATERIALS AND METHODS}

\section{Cells and Reagents}

HeLa cells were grown in DMEM supplemented with penicillin and streptomycin and $10 \%$ fetal bovine serum. Dig-TMP was prepared by conjugation of trimethylpsoralen (TMP) to digoxigenin as described previously (Thazhathveetil et al., 2007). Briefly, the 4 -chloromethyl derivative of TMP was reacted with 4,7,10-trioxa-1,13-tridecanediamine to give TMP with a soluble glycol amine sidechain. This was reacted with the digoxigenin $\mathrm{N}$-hydroxysuccinimide ester to give TMP linked to digoxigenin.

\section{Laser Localized ICLs}

A central cross was marked with a diamond pen on the growth surface of a 35-mm glass bottom culture dish $\left(\mathrm{MatTek}^{\mathrm{TM}}\right)$. The plates were also scribed on the side of the dish to provide an orientation mark. Cells were seeded so as to achieve $50-60 \%$ confluence at the time of an experiment. They were incubated with the $20 \mu \mathrm{M}$ Dig-TMP at $37^{\circ} \mathrm{C}$ for $20 \mathrm{~min}$ prior to photoactivation. Localized irradiation was performed using a Nikon Eclipse TE2000 confocal microscope with an SRS NL100 nitrogen laser-pumped dye laser (Photonics Instruments, St.
Charles, IL, USA). The $365 \mathrm{~nm}$ laser shoots 3 -ns pulses with a repetition rate of $10 \mathrm{~Hz}$. The power, measured at the back aperture of the $\times 60$ objective, was $0.7 \mathrm{nW}$. The laser was directed to deliver pulses to a specified rectangular ROI $(3$ pixel $\times 30$ pixel, $0.16 \mu \mathrm{m} / \mathrm{pixel}$ ) within the nucleus of a cell, visualized with a Plan Fluor $\times 60 / 1.25$ numerical aperture oil objective. The laser, controlled by Volocity- 5 software (PerkinElmer Life Sciences), was oriented by galvanometer-driven beam displacers and fired randomly throughout the region until the entire ROI was exposed. Throughout an experiment, cells were maintained at $37^{\circ} \mathrm{C}, 5 \% \mathrm{CO}_{2}$, and $80 \%$ humidity in an environmental chamber.

\section{Dig-TMP Photoactivation by UVA Lamp}

Cells were incubated with indicated concentrations of Dig-TMP, placed in a Rayonet chamber (Southern New England Ultraviolet Co.), and exposed to $365 \mathrm{~nm}$ light $\left(3 \mathrm{~J} / \mathrm{cm}^{2}\right)$. The temperature in the chamber was maintained at $37^{\circ} \mathrm{C}$. The cells were either fixed for immunostaining or returned to the incubator for the specified times.

\section{Immunostaining}

Fixed cells were permeabilized with $0.5 \%$ Triton X-100, $1 \%$ bovine serum albumin, $100 \mathrm{mM}$ glycine, and $0.2 \mathrm{mg} / \mathrm{ml}$ EDTA in PBS on ice for $10 \mathrm{~min}$. The cells were subsequently digested with RNase A in PBS-EDTA (5 mM) solution for $30 \mathrm{~min}$ at $37^{\circ} \mathrm{C}$. Cells were blocked in $10 \%$ goat serum in PBS and $0.01 \%$ sodium azide for $1 \mathrm{~h}$ at $37^{\circ} \mathrm{C}$ or overnight at $4^{\circ} \mathrm{C}$. For IF staining, cells were incubated with appropriate primary antibody diluted in blocking solution for $1 \mathrm{~h}$ at $37^{\circ} \mathrm{C}$. After three washes using $0.05 \%$ Tween 20 in PBS, cells were incubated with a corresponding fluorescence-tagged secondary antibody [Alexa Fluor goat antimouse or Alexa Fluor goat anti-rabbit (Invitrogen)]. After three washes, cells were mounted with ProLong Gold antifade reagent with 4',6-diamidino-2-phenylindole (Invitrogen). Stained cells were visualized and imaged using a Hamamatsu EM-CCD digital camera attached to the Nikon Eclipse TE2000 confocal microscope.

\section{DNA Fiber Analysis}

Cells were incubated for $24 \mathrm{~h}$ with $20 \mu \mathrm{M}$ CldU. They were then incubated with $20 \mu \mathrm{M}$ Dig-TMP for 20 min before UVA irradiation in a Rayonet chamber or exposure to the $365 \mathrm{~nm}$ laser in ROI in nuclei in cells in the immediate vicinity of the cross. Cells exposed to the UVA lamp were harvested by trypsinization and the cells were placed on a glass slide. Plates with cells containing laser localized ICLs were washed and a drop of trypsin from a drawn out capillary placed on the intersection of the cross. The detached cells were recovered and placed on a glass slide. Cells were then mixed with lysis buffer $(0.5 \%$ SDS in $200 \mathrm{mM}$ Tris- $\mathrm{HCl}$ [pH 7.5], $50 \mathrm{mM}$ EDTA) on the slide. After tilting, the slides were air-dried, fixed in 3:1 methanol/acetic acid, incubated in $2.5 \mathrm{M} \mathrm{HCl}$, neutralized in $0.4 \mathrm{M}$ Tris- $\mathrm{HCl}$ ( $\mathrm{pH}$ 7.5), washed in PBS, and immunostained. Antibodies and dilutions were rat anti-BrdU (CldU), 1:200; Dylight 649 goat anti-rat, 1:100; mouse anti-BrdU (IdU), 1:40 and chicken antidigoxigenin, 1:200; and Dylight 488 goat anti-mouse, 1:100 and Q dot 655 goat anti-chicken, 1:2,500. Imaging was carried out using 
A

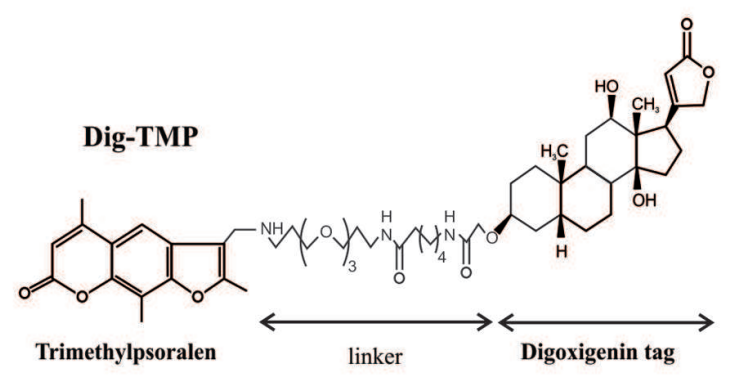

C

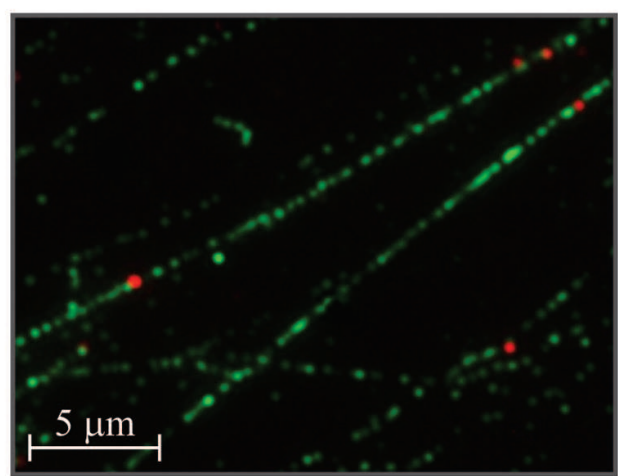

B

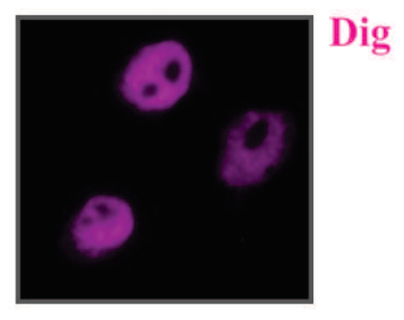

D

E

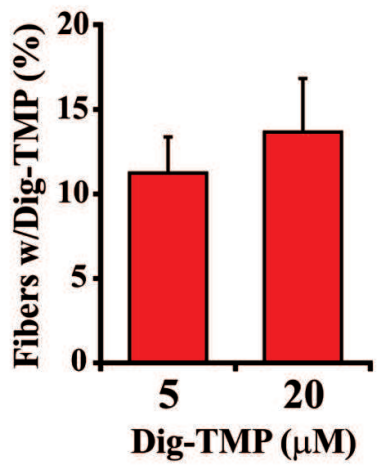

FIGURE 1 | Distribution of Dig-TMP on DNA fibers from cells after photoactivation by UVA lamp. (A) The structure of trimethylpsoralen (TMP) linked to Digoxigenin (Dig). (B) Cells were incubated with Dig-TMP and exposed to UVA in the Rayonet lamp chamber. They were then immunostained against the Dig tag. (C) Cells were prelabeled with CldU, then incubated with Dig-TMP and exposed to the UVA lamp. DNA fibers were prepared and immunostained for CldU (green) and Dig-TMP (red dots). (D) Cells were treated with the indicated concentration of Dig-TMP and DNA fibers prepared and stained, and fields photographed as in (C). The fraction of fibers in a field containing a Dig-TMP signal was determined. From cells treated with $5 \mu$ M Dig-TMP the number of fibers with a Dig signal and the total fibers scored in individual experiments were: 33 and 297; 25 and 279; 32 and 224. From cells treated with $20 \mu$ M Dig-TMP: 39 and $285 ; 21$ and 208; 35 and 206 (total fibers 742, 604). The Chi squared test showed no significant difference ( $p=0.114)$. (E) Fibers were prepared and stained from cells treated as in (C). The lengths of all fibers in a field, and the total number of Dig-TMP signals on fibers, were determined and used for the calculation of the number of Dig-TMP signals per $10^{3} \mathrm{~kb}$ of fiber. The number of Dig-TMP signals scored and the total number of fibers examined were: at $5 \mu \mathrm{M}-44$ and 78 ; 70 and 98 ; 54 and 65 ; at $20 \mu \mathrm{M}$ - 97 and $69 ; 81$ and $62 ; 80$ and $61(N=245,188)$. The $t$-test showed significant difference $(p<0.001)$ between the two means $(2.69,4.48, S D 0.095,0.285)$.

a Zeiss Axiovert $200 \mathrm{M}$ microscope with the AxioVision software packages (Zeiss). The quantum dot signal was imaged with a $\mathrm{Q}$ dot 655 filter.

\section{RESULTS}

\section{The Tag}

In order to develop an approach for imaging psoralen ICLs we had to attach a reliable and effective detection tag. In the first attempt, TMP was linked to rhodamine in an effort to generate a compound whose removal by repair could be visualized directly in live cells over time. Unfortunately, rhodamine is readily taken into mitochondria and little or no signal was observed in the nucleus. Another fluor, Oregon Green, was coupled to TMP. Although the Oregon Green compound did appear in the nucleus it was quickly bleached. Biotin psoralen conjugates have been used for many years to label DNA in vitro. However, biotin is a cofactor for carboxylases, with a strong cytoplasmic and mitochondrial presence. Like the rhodamine derivative very little of the biotin-TMP went to the nucleus. Finally, we synthesized the digoxigenin derivative (Figure 1A) (Thazhathveetil et al., 2007). Digoxigenin is a frequently used immuno-tag and commercial antibodies against it are available. Cells treated with the Dig-TMP and UVA and immunostained against the Dig tag displayed nuclear fluorescence. Therefore, Dig-TMP was used in all subsequent experiments.

An approach based on imaging a detection tag linked to psoralen adducts cannot distinguish the different products formed by the reaction of the compound with the genomic DNA. Since the goal of our studies was an understanding of how cells respond to ICLs it was of obvious importance to analyze the relative abundance of ICLs and single strand adducts (monoadducts) in genomic DNA following treatment of live cells with TMP/UVA, or Dig-TMP/UVA. DNA was purified from treated cells and subjected to nuclease P1 digestion and HPLC-tandem mass spectrometry. The results indicated that cell treatment with both TMP and Dig-TMP yielded ICL/monoadduct ratios of 10:1. That is, of 11 adducts, 10 were ICLs. Thus it was possible to interpret signals from the tagged 
A

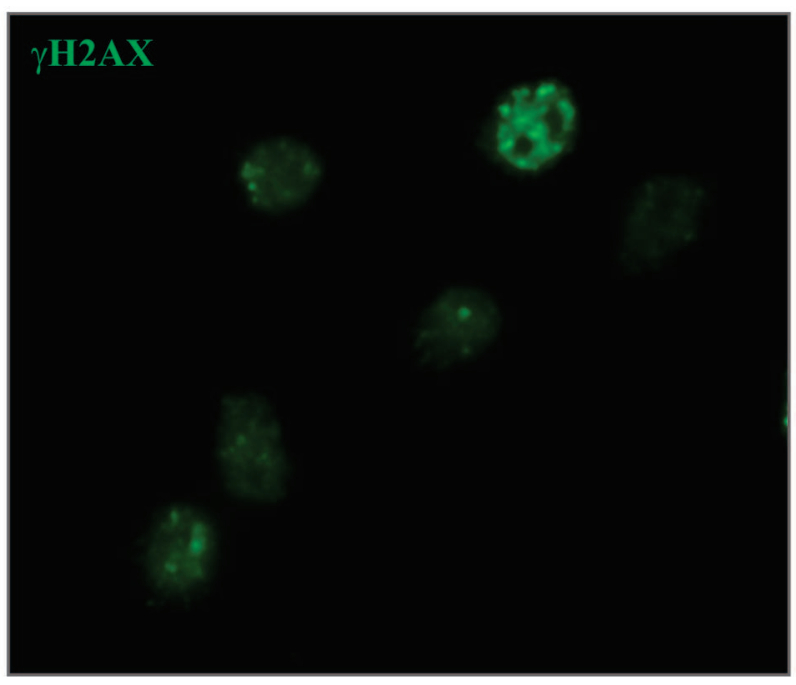

B

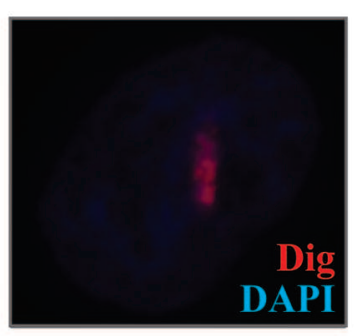

C

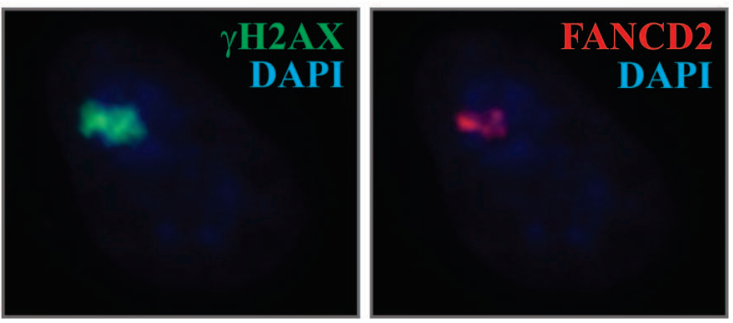

FIGURE 2 | Laser localized Dig-TMP induces the DDR. (A) Cells were incubated with $20 \mu$ M Dig-TMP and exposed to the UVA lamp. They were fixed and immunostained for $\gamma$-H2AX. (B) Cells were incubated with Dig-TMP and a defined region of interest was exposed to the 365 nm laser light. Cells were fixed and immunostained for the Dig tag. (C) Cells were incubated with Dig-TMP, exposed to the laser in an ROI and after 15 min fixed and immunostained for $\gamma$-H2AX (green) and FANCD2 (red).

psoralen as primarily derived from ICLs (see Huang et al., 2013 for detailed results of this analysis).

\section{Immunofluorescence of Dig-TMP}

Analysis of the Dig-TMP in cells following exposure to the UVA lamp revealed nuclear staining with the apparent exclusion from nucleoli (Figure 1B). This staining pattern did not permit any conclusion regarding the density or distribution of Dig-TMP ICls in genomic DNA. In order to address this question we took advantage of imaging technology based on DNA fibers. This approach is usually employed to address questions related to DNA replication. The methodology exploits the incorporation into DNA, during $\mathrm{S}$ phase, of nucleoside analogs that can be detected by IF using appropriate antibodies (for the halogenated analogs $\mathrm{CldU}$, IdU, or $\mathrm{BrdU}$ ) or by chemical conjugation with biotin (to EdU using click chemistry). The IF analyses illuminate tracks on individual DNA fibers stretched out on a glass surface (Schwab and Niedzwiedz, 2011).

Cells were incubated in medium containing CldU for $24 \mathrm{~h}$ to uniformly label DNA. They were then treated with $5 \mu \mathrm{M}$ Dig-TMP and UVA after which DNA fibers were spread on microscope slides and the CldU imaged by conventional IF. Individual Dig-TMP molecules covalently attached to the DNA were detected by immuno-quantum dot technology (Kad et al., 2010; Huang et al., 2013). A field with DNA fibers, some of which have Dig-TMP signals, is shown in Figure 1C. Analysis of the fibers indicated that about $10-15 \%$ had a Dig-TMP signal on them. The experiment was repeated with a higher concentration of Dig-TMP. Although the frequency of fibers with Dig-TMP signals increased slightly, the number of signals within fibers was clearly enhanced, such that the average distance between them was reduced (Figures 1D,E). Psoralen has been used as a probe of chromatin structure and DNA conformation (Wieshahn et al., 1977; Kouzine et al., 2013). We interpret our results as indicating that some regions of the genome are more accessible to the Dig-TMP than others, consistent with prior reports, and, at higher concentrations of compound, those regions are more extensively modified. However, it should be noted that we did not see the appearance of intense localized signals at the higher concentration, suggesting that the Dig-TMP molecules do not react at closely spaced hotspots, in contrast to the clustered breaks introduced by high-linear energy transfer (LET) ionizing radiation (Lorat et al., 2015).

\section{Laser Localized ICLs}

As shown above, treatment of cells with psoralen/UVA resulted in the introduction of ICLs throughout the nucleus. However, unlike the situation with DSBs, discrete foci of DDR proteins, such as $\gamma-\mathrm{H} 2 \mathrm{AX}$, did not form (Figure 2A). In this regard the treated cells are much like those exposed to UVC. So in order to "create" foci why not just employ the strategy of masking with a micropore filter, as was done successfully with UVC? Unfortunately, while the filters block shortwave UV, they do not block the long wave UV required for psoralen photoactivation. We attempted to construct effective filters by carbon or gold shadowing but were unsuccessful. Consequently, another approach was required.

In order to generate "foci" of ICLs and responding proteins, we employed laser localization. Cells were grown on a glass bottomed dish onto which a cross was marked with a diamond pen. The cells were incubated with Dig-TMP and ROI were exposed to $365 \mathrm{~nm}$ laser light. This produced a stripe of localized 
A

\section{Laser localized Dig-TMP induced ICLs}

- Label cells with CldU for 1 day

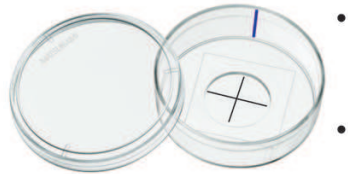

- Draw a cross on a glass bottom plate with a diamond pen

- Draw an orientation mark on plate wall (blue)

- Seed cells in the marked plate

- incubate ON in CldU

- Incubate cells in the presence of Dig-TMP

- Place plate in an environmental chamber in a confocal microscope equipped with a $365 \mathrm{~nm}$ laser

- Target a region of interest (red stripe) in the nuclei of $\sim 100$ cells around the cross (light blue area below). In these cells, in the targeted regions, the photoactivated Dig-TMP will generate ICLs.
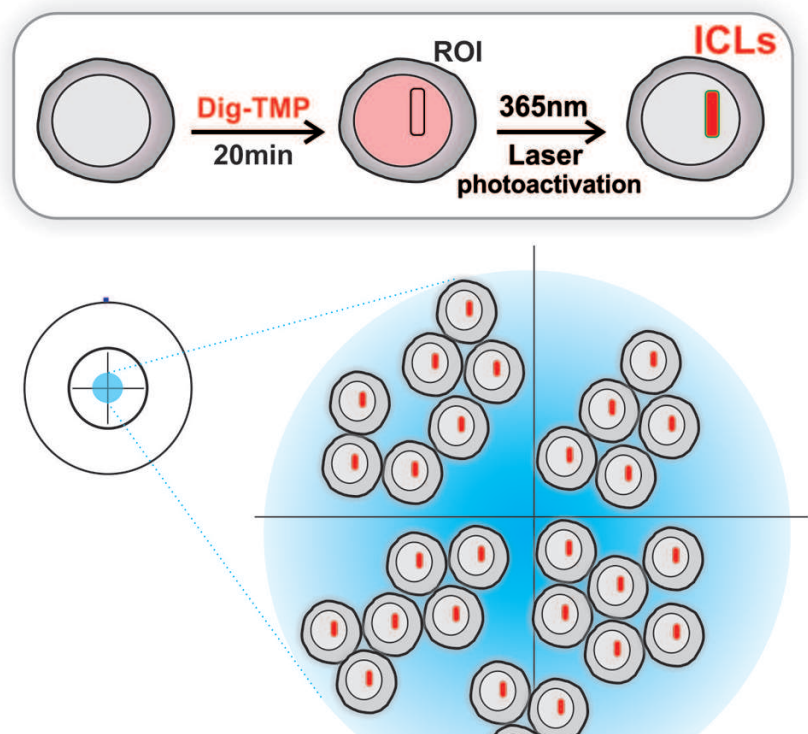

- Wash plate with PBS

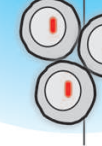

- Trypsinize the area around the center of the cross to recover cells targeted by the laser

- Proceed to stretch DNA fibers onto slides
B

\section{Dig-TMP/UVA lamp induced ICLs}

- Label cells with CldU for 2 days

- Incubate cells in the presence of Dig-TMP

- Place plate in Rayonet chamber and irradiate with UVA light. The whole nucleus of every cell in the plate is exposed to UVA light, which photoactivates Dig-TMP to induce the generation of ICLs throughout the genomic DNA.
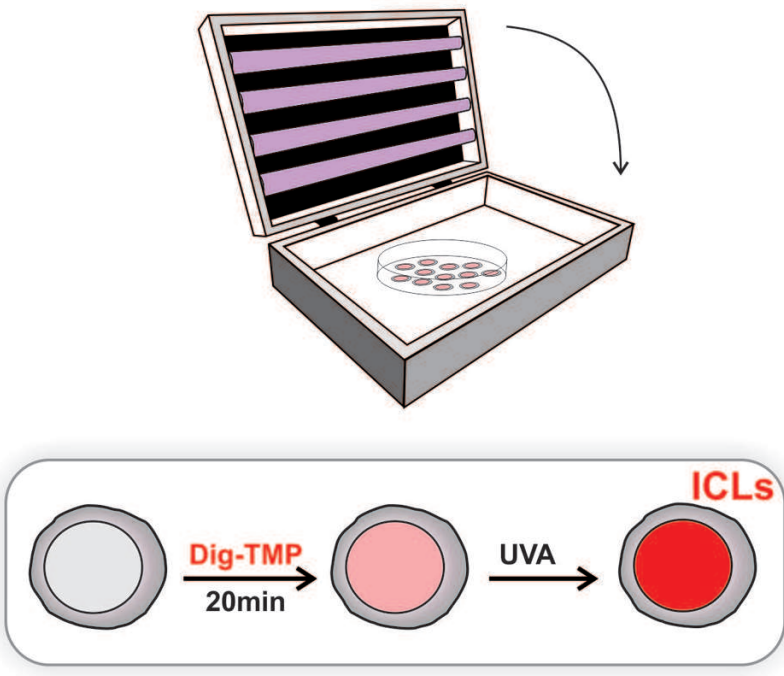

- Wash plate with PBS

- Trypsinize to recover cells

- Proceed to stretch DNA fibers onto slides

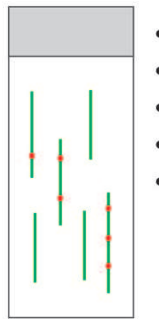

- Fix slides

- Stain for CldU and Dig

- Image

- Measure distance between Dig signals

- Compare

FIGURE 3 | Distribution of UVA lamp induced and laser localized ICLs. The experimental design is presented in the schematic. The ICLs, indicated in red, are localized to stripes after laser photoactivation of Dig-TMP at ROls (A), and distributed throughout the nucleus after exposure of cells to Dig-TMP and a UVA lamp (B). 
A

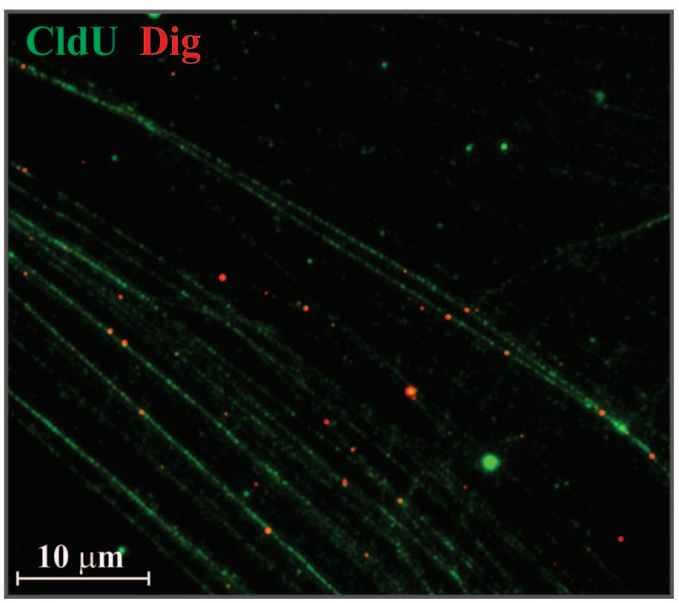

Dig-TMP / 365 nm LASER
B

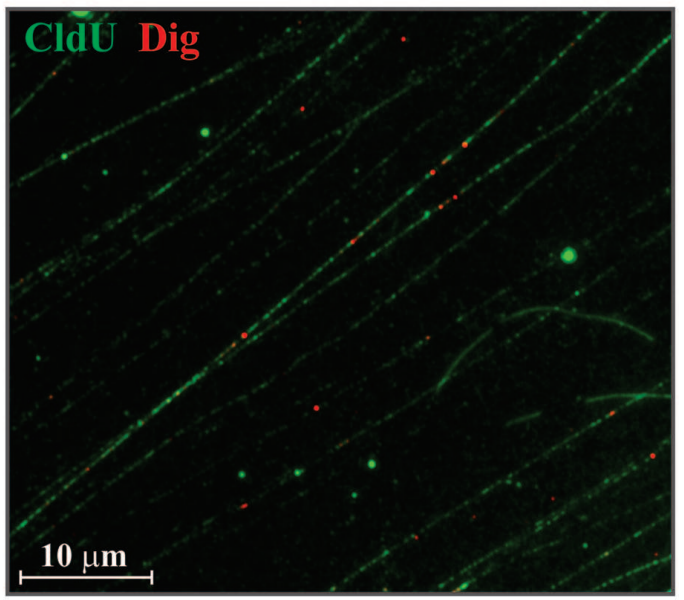

Dig-TMP / UVA lamp

C

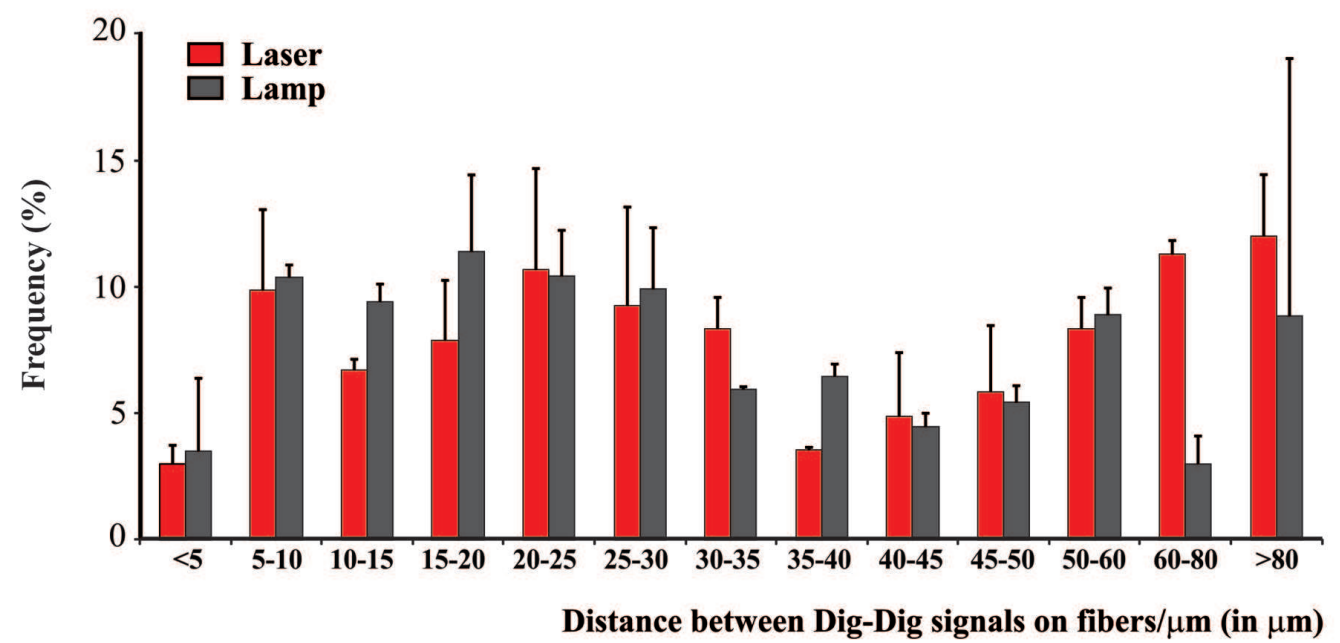

FIGURE 4 | The distribution of Dig-TMP ICLs on DNA molecules after photoactivation by laser or UVA lamp. (A) Cells were prelabeled with CldU, seeded in Mattek plates marked with a cross on the growth surface of the glass. The next day cells were incubated in $20 \mu \mathrm{M}$ Dig-TMP and the ROI in nuclei in cells close to the cross were photoactivated with the laser. Cells in the vicinity of the cross were harvested and placed on a slide and fibers spread, fixed, and immunostained for CldU (green) and Dig-TMP (red). (B) Cells were prelabeled with CldU, incubated with $20 \mu$ M Dig-TMP and exposed to the UVA lamp. (C) The distribution of Dig-TMP signals on fibers from cells with laser or lamp photoactivation. The plot represents results from 3 independent experiments (number of laser localized Dig-TMP signals used for distance measurements in the individual experiments-111, 85, 90; number of Dig-TMP signals induced by UVA lamp used for the measurements-102, 101, 102).

Dig-TMP adducts which could be displayed by IF against the Dig tag (Figure 2B) (Thazhathveetil et al., 2007). The location of individual treated cells was referenced to the cross mark.

The laser localized ICLs were inducers of the DDR, demonstrated by the accumulation in the stripes of $\gamma-\mathrm{H} 2 \mathrm{AX}$ and the Fanconi Anemia protein FANCD2 (Yan et al., 2012) (Figure 2C). We have used this approach to detail the recruitment of many $\mathrm{DDR}$ and repair proteins to the ICLs (Muniandy et al., 2009; Xu et al., 2010; Yan et al., 2010, 2012; McNeill et al., 2013; Singh et al., 2013; Jiang et al., 2015; Tian et al., 2015). This requires the combination of both compound and light. In the absence of psoralen, exposure to the laser at the intensity used in these experiments does not induce the recruitment of the DDR and repair proteins.

\section{Distribution of Laser Localized ICLs in Genomic DNA}

The laser and the UVA lamp are quite different light sources for psoralen photoactivation. Since both are used in experiments to introduce psoralen ICLs it was important to ask if the 


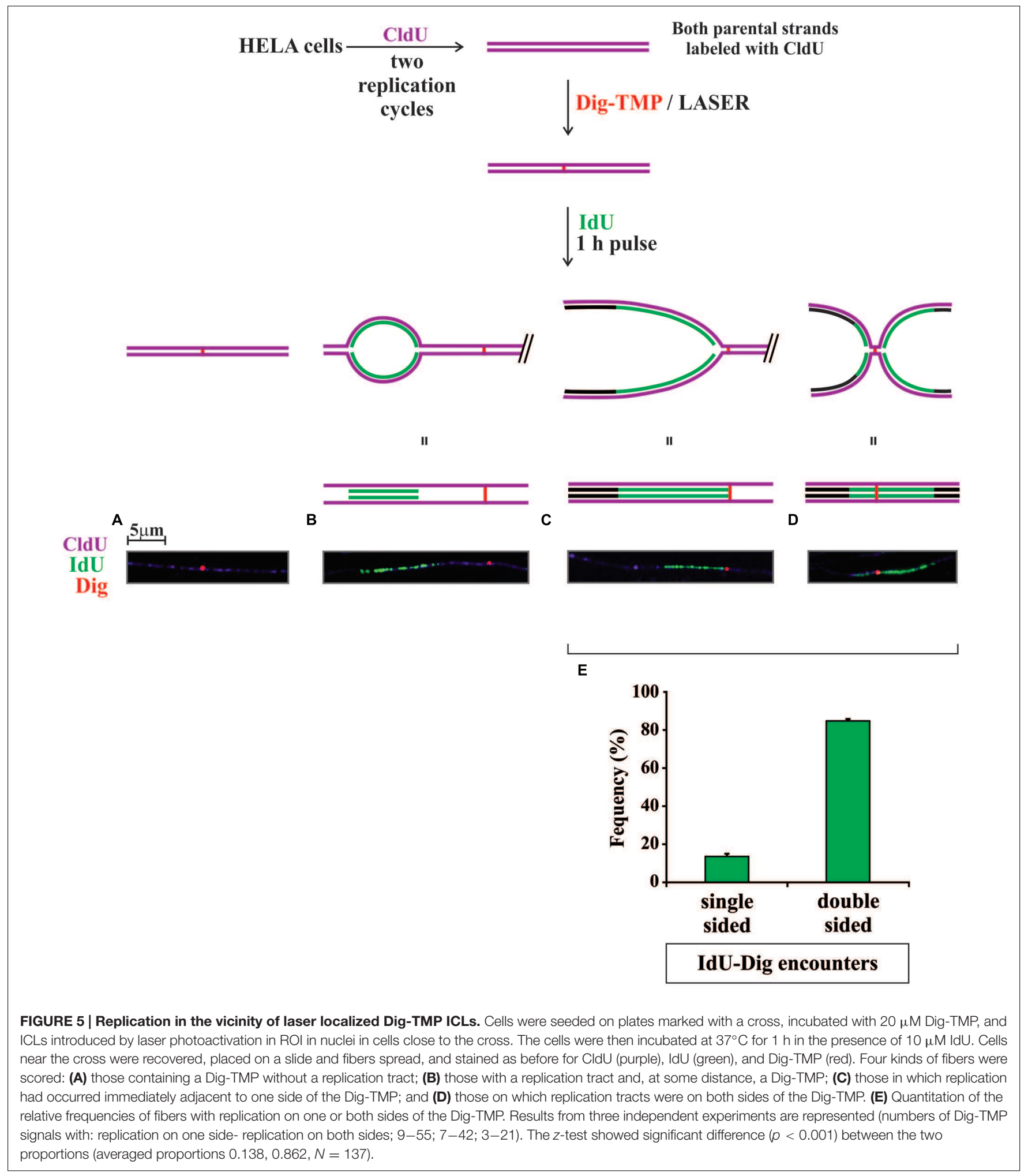

distribution of laser localized Dig-TMP ICLs in genomic DNA differed markedly from that in experiments with the UVA lamps. Our approach to answering these questions is diagrammed in Figure 3. Cells were incubated with $\mathrm{CldU}$ for $24 \mathrm{~h}$ to uniformly label DNA. They were then incubated in $20 \mu \mathrm{M}$ Dig-TMP and laser localized ICLs were introduced into ROI in nuclei in cells located close to the cross. Those cells were harvested and DNA fibers were prepared (Figure 3A). In parallel, fibers 
were spread from cells incubated with the same concentration of Dig-TMP and exposed to the UVA lamps (Figure 3B). The Dig-TMP adducts were displayed by the immuno-quantum dot technique (Figures 4A,B). The distribution of Dig-TMP signals on individual fibers from both preparations was determined (Figure 4C). These measurements demonstrated very little difference in Dig-TMP adduct spacing between the two samples. This argued that the distribution of TMP adducts was based on the properties of the psoralen, not the light source.

\section{Replication Fork Encounters with Laser Localized ICLs}

In previous work, we combined DNA fiber technology and immuno-quantum dot imaging in a study of replication fork encounters with Dig-TMP ICLs introduced by exposure to a UVA lamp (Huang et al., 2013). We observed patterns in which replication tracts terminated at ICLs (single sided), as well as those in which replication occurred on both sides of an ICL (double sided). It was of interest to determine the relevance of this observation to events in the stripes containing the laser localized ICLs. Given the obvious differences in light source the immediate question was whether or not there would be replication on fibers containing ICLs induced by the laser.

The experimental approach is diagrammed in Figure 5. Cells were incubated with CldU for $24 \mathrm{~h}$ to prelabel cellular DNA. The medium was changed, and they were incubated with DigTMP. Then ROI in nuclei in cells close to the cross were exposed to the laser. The medium was changed to one containing IdU and the cells returned to the incubator for $1 \mathrm{~h}$. Cells proximal to the cross were harvested as before (Figure 3A), DNA fibers spread, and the CldU prelabel, the IdU replication label, and the Dig-TMP displayed. The prelabel made it possible to trace the continuity of fibers over much greater length than possible if only replication tracts had been displayed. We observed fibers with Dig-TMP spots, but no replication tracts on the fiber (Figure 5A). Replication tracts were observed on fibers at various distances from Dig-TMP signals (Figure 5B), indicating that there was indeed replication on fibers isolated from the ROI exposed to the laser. In addition there were tracts terminated by Dig-TMP spots, as well as those with embedded Dig-TMP signals (Figures 5C,D). The relative frequencies of each, $15 \%$ single sided, $85 \%$ double sided (Figure 5E), were very similar to what had been observed previously in experiments in which photoactivation was with the lamp (Huang et al., 2013). These results indicated that replication occurred in similar fashion in the vicinity of Dig-TMP ICLs regardless of the UVA source.

We conclude from these experiments that the fundamentals regarding psoralen ICL formation driven by the UVA lamp and laser are quite similar such that results from the one approach can inform the other.

\section{REFERENCES}

Chalfie, M., Tu, Y., Euskirchen, G., Ward, W. W., and Prasher, D. C. (1994). Green fluorescent protein as a marker for gene expression. Science 263, 802-805. doi: $10.1126 /$ science. 8303295

\section{DISCUSSION}

Laser induced DNA damage is the basis of a substantial literature on the induction of the DDR and the repair of DSBs as monitored by surrogate markers. As noted above, the difficulty of direct identification of breaks precludes assessment of lesion frequency and spacing in the ROI. Furthermore, the possibility of extensive damage due to exposure of DNA to the high intensities associated with lasers has been a source of concern (Williams et al., 2007). In this communication, we have resolved these issues in regard to the psoralen ICLs. Our experiments indicate that the density of psoralen DNA photoproducts is similar regardless of the light source for photoactivation. Consequently, it is reasonable to extrapolate results and conclusions from experiments with one UVA source to experiments with the other. Furthermore, the demonstration of replication tracts in the immediate vicinity of the psoralen adducts argues that replication fork encounters with laser localized ICLs do occur, with a pattern distribution quite like that observed with UVA lamp induced psoralen ICLs. These observations enable experiments designed to examine replication fork dynamics in the vicinity of the laser activated psoralen ICLs. Such experiments are not possible with laser induced DSBs, because there is no methodology for direct detection of the breaks.

The laser localization strategy obviously requires photodependent adduct formation, and so is limited to appropriate compounds. However, display of DNA damage on DNA fibers requires only a DNA adduct detectable by chemical conjugation or immunological reagents. For example, a DNA reactive agent linked to one of the partners in azide/alkyne click chemistry would be applicable. Similarly, antibodies or specific binding proteins against DNA adducts (carcinogens, UV photoproducts) could also be employed (Poirier, 1993; Dreze et al., 2014; McCready, 2014). Thus, we think it likely that the fiber strategies can be generalized to other DNA damaging agents, and could be used to address question regarding adduct distribution, repair, and replication fork encounters.

\section{AUTHOR CONTRIBUTIONS}

JH, HG, MP, PM, JG, MAB, and MMS participated in designing and performing experiments, analyzing results and contributed to generating the figures and writing the paper.

\section{FUNDING}

This research was supported in part by the Intramural Research Program of the NIH, National Institute on Aging (Z01 AG000746-08) and the Fanconi Anemia Research Fund.

Ciccia, A., and Elledge, S. J. (2010). The DNA damage response: making it safe to play with knives. Mol. Cell 40, 179-204. doi: 10.1016/j.molcel.2010.09.019

Dinant, C., de, J. M., Essers, J., van Cappellen, W. A., Kanaar, R., Houtsmuller, A. B., et al. (2007). Activation of multiple DNA repair pathways by sub-nuclear damage induction methods. J. Cell Sci. 120, 2731-2740. doi: 10.1242/jcs.004523 
Dreze, M., Calkins, A. S., Galicza, J., Echelman, D. J., Schnorenberg, M. R., Fell, G. L., et al. (2014). Monitoring repair of UV-induced 6-4photoproducts with a purified DDB2 protein complex. PLoS ONE 9:e85896. doi: 10.1371/journal.pone.0085896

Dronkert, M. L., and Kanaar, R. (2001). Repair of DNA interstrand cross-links. Mutat. Res. 486, 217-247. doi: 10.1016/S0921-8777(01)00092-1

Hlavin, E. M., Smeaton, M. B., Noronha, A. M., Wilds, C. J., and Miller, P. S. (2010). Cross-link structure affects replication-independent DNA interstrand cross-link repair in mammalian cells. Biochemistry 49, 3977-3988. doi: $10.1021 / \mathrm{bi} 902169 \mathrm{q}$

Huang, J., Liu, S., Bellani, M. A., Thazhathveetil, A. K., Ling, C., de Winter, J. P., et al. (2013). The DNA translocase FANCM/MHF promotes replication traverse of DNA interstrand crosslinks. Mol. Cell 52, 434-446. doi: 10.1016/j.molcel.2013.09.021

Jiang, Q., Paramasivam, M., Aressy, B., Wu, J., Bellani, M., Tong, W., et al. (2015). MERIT40 cooperates with BRCA2 to resolve DNA interstrand cross-links. Genes Dev. 29, 1955-1968. doi: 10.1101/gad.264192.115

Kad, N. M., Wang, H., Kennedy, G. G., Warshaw, D. M., and Van Houten, B. (2010). Collaborative dynamic DNA scanning by nucleotide excision repair proteins investigated by single- molecule imaging of quantum-dot-labeled proteins. Mol. Cell 37, 702-713. doi: 10.1016/j.molcel.2010.02.003

Knappik, A., and Pluckthun, A. (1994). An improved affinity tag based on the FLAG peptide for the detection and purification of recombinant antibody fragments. Biotechniques 17, 754-761.

Knipscheer, P., Raschle, M., Scharer, O. D., and Walter, J. C. (2012). Replicationcoupled DNA interstrand cross-link repair in Xenopus egg extracts. Methods Mol. Biol. 920, 221-243. doi: 10.1007/978-1-61779-998-3_16

Kolb, H. C., Finn, M. G., and Sharpless, K. B. (2001). Click chemistry: diverse chemical function from a few good reactions. Angew. Chem. Int. Ed. Engl. 40, 2004-2021. doi: 10.1002/1521-3773(20010601)40:11<2004::AIDANIE2004> 3.0.CO;2-5

Kouzine, F., Gupta, A., Baranello, L., Wojtowicz, D., Ben-Aissa, K., Liu, J., et al. (2013). Transcription-dependent dynamic supercoiling is a short-range genomic force. Nat. Struct. Mol. Biol. 20, 396-403. doi: 10.1038/nsmb.2517

Lai, C., Cao, H., Hearst, J. E., Corash, L., Luo, H., and Wang, Y. (2008). Quantitative analysis of DNA interstrand cross-links and monoadducts formed in human cells induced by psoralens and UVA irradiation. Anal. Chem. 80, 8790-8798. doi: $10.1021 /$ ac $801520 \mathrm{~m}$

Lan, L., Nakajima, S., Oohata, Y., Takao, M., Okano, S., Masutani, M., et al. (2004). In situ analysis of repair processes for oxidative DNA damage in mammalian cells. Proc. Natl. Acad. Sci. U.S.A. 101, 13738-13743. doi: 10.1073/pnas.0406048101

Lorat, Y., Brunner, C. U., Schanz, S., Jakob, B., Taucher-Scholz, G., and Rube, C. E. (2015). Nanoscale analysis of clustered DNA damage after high-LET irradiation by quantitative electron microscopy-the heavy burden to repair. DNA Repair (Amst.) 28, 93-106. doi: 10.1016/j.dnarep.2015.01.007

McCready, S. (2014). An immunoassay for measuring repair of UV photoproducts. Methods Mol. Biol. 1105, 551-564. doi: 10.1007/978-1-62703-739-6_38

McNeill, D. R., Paramasivam, M., Baldwin, J., Huang, J., Vyjayanti, V. N., Seidman, M. M., et al. (2013). NEIL1 responds and binds to psoraleninduced DNA interstrand crosslinks. J. Biol. Chem. 288, 12426-12436. doi: 10.1074/jbc.M113.456087

Miller, M. A., Kolb, P. E., and Raskind, M. A. (1993). A method for simultaneous detection of multiple mRNAs using digoxigenin and radioisotopic cRNA probes. J. Histochem. Cytochem. 41, 1741-1750. doi: 10.1177/41.12.8245421

Muniandy, P. A., Liu, J., Majumdar, A., Liu, S. T., and Seidman, M. M. (2010). DNA interstrand crosslink repair in mammalian cells: step by step. Crit. Rev. Biochem. Mol. Biol. 45, 23-49. doi: 10.3109/10409230903501819

Muniandy, P. A., Thapa, D., Thazhathveetil, A. K., Liu, S. T., and Seidman, M. M. (2009). Repair of laser-localized DNA interstrand cross-links in G1 phase mammalian cells. J. Biol. Chem. 284, 27908-27917. doi: 10.1074/jbc.M109.029025

Nerurkar, L. S., Namba, M., Brashears, G., Jacob, A. J., Lee, Y. J., and Sever, J. L. (1984). Rapid detection of herpes simplex virus in clinical specimens by use of a capture biotin-streptavidin enzyme-linked immunosorbent assay. J. Clin. Microbiol. 20, 109-114.

Poirier, M. C. (1993). Antisera specific for carcinogen-DNA adducts and carcinogen-modified DNA: applications for detection of xenobiotics in biological samples. Mutat. Res. 288, 31-38. doi: 10.1016/0027-5107(93)90205-T
Raschle, M., Knipsheer, P., Enoiu, M., Angelov, T., Sun, J., Griffith, J. D., et al. (2008). Mechanism of replication-coupled DNA interstrand crosslink repair. Cell 134, 969-980. doi: 10.1016/j.cell.2008.08.030

Rogakou, E. P., Boon, C., Redon, C., and Bonner, W. M. (1999). Megabase chromatin domains involved in DNA double-strand breaks in vivo. J. Cell Biol. 146, 905-916. doi: 10.1083/jcb.146.5.905

Rohleder, F., Huang, J., Xue, Y., Kuper, J., Round, A., Seidman, M., et al. (2016). FANCM interacts with PCNA to promote replication traverse of DNA interstrand crosslinks. Nucleic Acids Res. 44, 3219-3232. doi: 10.1093/nar/gkw037

Rothkamm, K., Barnard, S., Moquet, J., Ellender, M., Rana, Z., and BurdakRothkamm, S. (2015). DNA damage foci: meaning and significance. Environ. Mol. Mutagen. 56, 491-504. doi: 10.1002/em.21944

Schwab, R. A., and Niedzwiedz, W. (2011). Visualization of DNA replication in the vertebrate model system DT40 using the DNA fiber technique. J. Vis. Exp. 56:e3255. doi: 10.3791/3255

Sengerova, B., Allerston, C. K., Abu, M., Lee, S. Y., Hartley, J., Kiakos, K., et al. (2012). Characterization of the human SNM1A and SNM1B/Apollo DNA repair exonucleases. J. Biol. Chem. 287, 26254-26267. doi: 10.1074/jbc.M112.367243

Shen, X., Do, H., Li, Y., Chung, W. H., Tomasz, M., de Winter, J. P., et al. (2009). Recruitment of fanconi anemia and breast cancer proteins to DNA damage sites is differentially governed by replication. Mol. Cell 35, 716-723. doi: 10.1016/j.molcel.2009.06.034

Singh, T. R., Ali, A. M., Paramasivam, M., Pradhan, A., Wahengbam, K., Seidman, M. M., et al. (2013). ATR-dependent phosphorylation of FANCM at serine 1045 is essential for FANCM functions. Cancer Res. 73, 4300-4310. doi: 10.1158/0008-5472.CAN-12-3976

Thazhathveetil, A. K., Liu, S. T., Indig, F. E., and Seidman, M. M. (2007). Psoralen conjugates for visualization of genomic interstrand cross-links localized by laser photoactivation. Bioconjug. Chem. 18, 431-437. doi: 10.1021/bc060309t

Tian, Y., Paramasivam, M., Ghosal, G., Chen, D., Shen, X., Huang, Y., et al. (2015). UHRF1 contributes to DNA damage repair as a lesion recognition factor and nuclease scaffold. Cell Rep. 10, 1957-1966. doi: 10.1016/j.celrep.2015. 03.038

Volker, M., Mone, M. J., Karmakar, P., van, H. A., Schul, W., Vermeulen, W., et al. (2001). Sequential assembly of the nucleotide excision repair factors in vivo. Mol. Cell 8, 213-224. doi: 10.1016/S1097-2765(01)00281-7

Wieshahn, G. P., Hyde, J. E., and Hearst, J. E. (1977). The photoaddition of trimethylpsoralen to Drosophila melanogaster nuclei: a probe for chromatin substructure. Biochemistry 16, 925-932. doi: 10.1021/bi00 $624 \mathrm{a} 018$

Williams, E. S., Stap, J., Essers, J., Ponnaiya, B., Luijsterburg, M. S., Krawczyk, P. M., et al. (2007). DNA double-strand breaks are not sufficient to initiate recruitment of TRF2. Nat. Genet. 39, 696-698. doi: 10.1038/ng0607-696

Xu, D., Muniandy, P., Leo, E., Yin, J., Thangavel, S., Shen, X., et al. (2010). Rif1 provides a new DNA-binding interface for the Bloom syndrome complex to maintain normal replication. EMBO J. 29, 3140-3155. doi: 10.1038/emboj.2010.186

Yan, Z., Delannoy, M., Ling, C., Daee, D., Osman, F., Muniandy, P. A., et al. (2010). A histone-fold complex and FANCM form a conserved DNAremodeling complex to maintain genome stability. Mol. Cell 37, 865-878. doi: 10.1016/j.molcel.2010.01.039

Yan, Z., Guo, R., Paramasivam, M., Shen, W., Ling, C., Fox, D., et al. (2012). A ubiquitin-binding protein, FAAP20, links RNF8-mediated ubiquitination to the Fanconi anemia DNA repair network. Mol. Cell 47, 61-75. doi: 10.1016/j.molcel.2012.05.026

Conflict of Interest Statement: The authors declare that the research was conducted in the absence of any commercial or financial relationships that could be construed as a potential conflict of interest.

Copyright (C) 2016 Huang, Gali, Paramasivam, Muniandy, Gichimu, Bellani and Seidman. This is an open-access article distributed under the terms of the Creative Commons Attribution License (CC BY). The use, distribution or reproduction in other forums is permitted, provided the original author(s) or licensor are credited and that the original publication in this journal is cited, in accordance with accepted academic practice. No use, distribution or reproduction is permitted which does not comply with these terms. 